\title{
Multiple Attribute Decision Making Based Relay Vehicle Selection for Electric Vehicle Communication
}

\author{
Qiang Zhao ${ }^{1,2}$, Yaoyao $\mathrm{Wu}^{2}$ \\ ${ }^{1}$ Northeastern University at Qinhuangdao, China \\ ${ }^{2}$ Northeastern University, China
}

\begin{abstract}
Large-scale electric vehicle integration into power grid and charging randomly will cause serious impacts on the normal operation of power grid. Therefore, it is necessary to control the charging behavior of electric vehicle, while information transmission for electric vehicle is significant. Due to the highly mobile characteristics of vehicle, transferring information to power grid directly might be inaccessible. Relay vehicle (RV) can be used for supporting multi-hop connection between SV and power grid. This paper proposes a multiple attribute decision making (MADM)-based RV selection algorithm, which considers multiple attribute, including data transfer rate, delay, route duration. It takes the characteristics of electric vehicle communication into account, which can provide protection for the communication services of electric vehicle charging and discharging. Numerical results demonstrate that compared to previous algorithm, the proposed algorithm offer better performance in terms of throughput, transmission delay.
\end{abstract}

\section{Introduction}

As the energy crisis and environmental pollution becoming more and more se-rious, electric vehicles (EV) are used more widely as an important means to resolve the crisis [1]. However large-scale EV integration into power grid and charging randomly will cause serious impacts on the normal operation of power grid. Therefore, it is necessary to control the charging behavior of EV by communicating with power grid, obtaining the status, the battery remaining amount, location, speed and other information of $\mathrm{EV}$ can assist us to schedule their provisioning properly.

Information transmission network structure of electric vehicle accessing to power grid consist of on board unit(OBU), Road side unit(RSU), data processing center and Dispatch center. Vehicle manufactures have been equipped with navigation and infotainment equipment for the electric vehicle, and wireless technology to support vehicle to vehicle and vehicle to RSU communication which provided by, e.g. IEEE $802.11 \mathrm{p}$, vehicle ad hoc network(VANET) [2]. VANET provide communications among nearby vehicles and nearby fixed roadside equipment [3]. And it has many own characteristics. VANET forms a dynamic topology due to the high speed of the vehicles and requires real time packet transfer; the node of VANET is regular in the sense because the vehicles move on the fixed roadways at high speeds; channel fading property; channel bandwidth limited severely. These characteristics pose various challenges in choosing optimal RV.
The problem of RV selection for VANET has been studied in previous literatures. In [4], a relay selection algorithm based on link stability use link duration (Link Expiration Time, LET) and route duration (Route Expiration Time, RET) to characterize the stability of link between vehicles. In [5], the authors propose a new RV selection algorithm which selects the minimum load RVs to result in the load balancing. In [6], based on the position of vehicles and IEEE $802.16 \mathrm{j}$, the authors developed an analytical model for locating and selecting the optimal RV, which can get higher capacity. Reference [7] proposes a decentralized joint relay selection and power allocation algorithm for a wireless relay network based on coalitional Game theory. Reference [8] considers the various attributes of candidate RV, e.g. moving speed, the received signal strength (RSS), RET between SV and RV, and chooses the optimal relay based on the maximum weighted value.

In this paper, a RV selection based on multiple attribute decision making is proposed. The advantage of this decision making technique is that it considers multiple attribute, which can take many aspects of system into account such as data transmission rate and delay which characterize real-time, RET characterize the link stability.

The rest of this paper is organized as follows. The proposed scheme is presented in the Section 2. Multiattribute decision is used to solve the optimization problem in Section 3. Numerical results are given in Section 4. Finally, we conclude the paper in Section 5.

\footnotetext{
${ }^{\mathrm{a}}$ Corresponding author:yhhan723@126.com
} 


\section{System model}

In this section, the network model and channel model we consider in this paper are described.

\subsection{Network model}

In this paper, a VANET application scenario including of multiple SVs, multiple RVs, and one RSU is considered, as shown in Figure. 1. VANETs provide communication between OBUs in nearby vehicles, and between OBUs in vehicles and RSUs, which are fixed equipment located on the road. When $\mathrm{SV}$ is out of the communication range of $\mathrm{RSU}, \mathrm{RV}$ is referred to as a particular type of vehicles which are capable of offering relaying functions to vehicles. For convenience, in this paper, we consider only two-hop connections from the SV to the RSU.

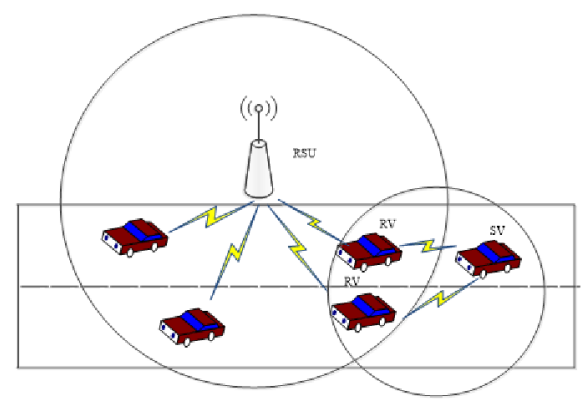

Figure 1. Network Model

\subsection{Channel model}

In this paper, considering the high speed mobility characteristic of $\mathrm{EV}$, the communication channel in VANET is modeled as Nakagami fading channel with the channel characteristics h1 following the probability distribution function (pdf) [9]:

$$
f\left(h_{1}\right)=\frac{2 m^{m}}{\Omega(d)^{m} \Gamma(m)} h_{1}^{2 m-1} \exp \left(-\frac{m}{\Omega(d)} h_{1}^{2}\right)
$$

Where $\boldsymbol{m}$ is the Nakagami fading parameter $(\boldsymbol{m} \geq \mathbf{l} / \mathbf{2})$, and $\boldsymbol{\Gamma}(\cdot)$ is the gamma function $[10], \mathbf{\Omega}(d)$ denotes power loss due to transmission distance $d$, and can be expressed as [11]:

$$
\Omega(d)=\frac{P_{t} G_{t} G_{r} h_{t}^{2} h_{r}^{2}}{d^{\theta} L}
$$

Where $\boldsymbol{P}_{\boldsymbol{t}}$ is the transmission power, $\boldsymbol{G}_{\boldsymbol{t}}$ and $\boldsymbol{G}_{\boldsymbol{r}}$ are antenna gains of the transmitter and receiver, respectively, $\boldsymbol{h}_{\boldsymbol{t}}$ and $\boldsymbol{h}_{\boldsymbol{r}}$ are the antenna heights of transmitter and receiver, respectively, $\boldsymbol{\theta}$ is the path loss exponent, and $L$ is the system loss.

The channel between SV and RV can be modeled as a cascaded Nakagami fading channel with the number of cascade being 2 and follow the channel pdf as [11]:

$$
f\left(h_{2}\right)=\frac{2}{h_{2} \Gamma\left(m_{1}\right) \Gamma\left(m_{2}\right)} G_{0,2}^{2,0}\left[\left.\frac{m_{1} m_{2} h_{2}^{2}}{\Omega_{1} \Omega_{2}}\right|_{m_{1}, m_{2}} ^{-}\right]
$$

Where, $\boldsymbol{G}_{\mathbf{0}, \mathbf{2}}^{\mathbf{2 0} \mathbf{0}}$ is the Meijer G-function [10], $\boldsymbol{\Omega}_{\boldsymbol{I}}=\boldsymbol{E}\left[\boldsymbol{h}_{\boldsymbol{f}}^{\mathbf{2}}\right]$ and $\boldsymbol{m}_{t}=\mathbf{\Omega}_{l}^{2} / E\left(\left(h_{l} \Omega_{l}\right)^{2}\right) \geq \mathbf{1} / 2, l=1,2$, The SNR can be calculated as $\boldsymbol{E}\left(\boldsymbol{h}^{2}\right) \frac{E_{s}}{N}$, where $\boldsymbol{h}$ is the gain of associated channel, $\boldsymbol{E}_{\boldsymbol{x}}$ is the alerage transmission power, $\boldsymbol{N}_{\mathrm{0}}$ is the power of noise.

\section{The RV Selection algorithm}

In this section, a utility based RV selection algorithm is proposed for VANET.

\subsection{Basic idea}

In this paper, the proposed RV selection algorithm involve four steps: (1) SV receive the RSS, the available bandwidth and connection delay from RSU, and determine whether need RV; (2) when the SV cannot transfer the data to the RSU directly, SV will broadcast the information containing the user service requirements of bandwidth, delay and others, and request to obtain the available information of neighbor RV; (3) if the condition qualify the demand of $\mathrm{SV}, \mathrm{RV}$ should send the information to $\mathrm{SV}$ which include location information, bandwidth, delay; (4) based on the information from RV, SV determine the candidate RVs; (5) finally, according to the multi attribute optimization RV selection algorithm, calculate the utility function of RVs and choose optimal RV.

\subsection{Multiple attribute decision making (MADM)}

Place the figure as close as possible after the point where it is first referenced in the text. If there is a large number of figures and tables it might be necessary to place some before their text citation. If a figure or table is too large to fit into one column, it can be centred across both columns at the top or the bottom of the page.

The relay selection function can be seen as an optimization problem, where the goal is to selection an optimal relay that guarantees information sent to RSU and maximizes the performance of system. The MADM model provides a solution to the problem of choosing an alternative from a finite set of options characterized in terms of their attributes. Multiple attribute decision making method mainly includes three steps: normalization method for attribute ratings, attribute weighting and comprehensive ranking scheme [12].

1. Normalization method for attribute ratings

Different units may be used for comparing attributes which cause computational problems. Therefore, optimal normalization techniques are necessary to perform interattribute and intra-attribute comparisons. Attributes can be divided into two types: benefit attributes and cost attributes [13]:

In this paper, the communication between EV and RSU need to provide high-bandwidth, low-latency messages, so ,we consider the factors which affect the performance of relay, such as data transmission rate, delay, the stability between SV and relay link .

- Normalization of data transmission rate 
The data transmission rate between SV and RSU express as:

$$
R_{S-R S U}=\min \left(\mathrm{R}_{S-i}, \mathrm{R}_{i-R S U}\right)
$$

Where, $R_{S-i}$ is the data transmission rate between $\mathrm{SV}$ and candidate RV, $R_{i-R S U}$ is the data transmission rate between RV and RSU. And according to the Shannon equation, they can express as:

$$
\begin{aligned}
R_{S-i} & =B^{R} \log _{2}\left(1+\gamma_{S-i}\right) \\
R_{i-R S U} & =B^{R} \log _{2}\left(1+\gamma_{i-R S U}\right)
\end{aligned}
$$

Where, $\gamma_{S-i}$ and $\gamma_{i-R S U}$ denote the signal to noise ratio(SNR) of link between the SV and the $i$ th candidate $\mathrm{RS}$, and from $i$ th candidate RS to RSU, respectively.

Normalization data transmission rate express as :

$$
l_{i 1}^{V}=\frac{R_{S-R S U}}{\max \left(R_{S-i}, R_{i-R S U}\right)}
$$

- Normalization of delay

We assume there is two-hop connection from the SV to the RSU, so the connection delay for SV to access RSU equals the sum of the connection delays corresponding to each link. The normalized total connection delay can be expressed as:

$$
l_{i 2}^{V}=\bar{D}_{s, y_{i}}+\bar{D}_{V_{i}, R}
$$

Where, $\bar{D}_{s, v_{i}}$ and $\bar{D}_{v_{i}, R}$ denote the normalized connection delay for the SV to access to the $i$ th candidate $\mathrm{RV}$, and for the $i$ th candidate RV to access the RSU, respectively, and can be expressed as follows:

$$
\begin{gathered}
\bar{D}_{S, V_{i}}=\frac{D_{r e q}^{\max }-D_{S, V_{i}}}{D_{r e q}^{\max }-D_{r e q}^{\min }} \\
\bar{D}_{V_{i}, R}=\frac{D_{V_{i}, R}^{\max }-D_{V_{i}, R}}{D_{V_{i}, R}^{\max }-D_{V_{i}, R}^{\min }}
\end{gathered}
$$

Where, $D_{S, V_{i}}$ and $D_{V_{i}, R}$, denote the connection delay of the $i$ th candidate RV and the RSU, respectively, $D_{V_{i}, R}^{\min }, D_{V_{i}, R}^{\max }$ define the minimum and maximum connection delay required for the RS to access to the infrastructure.

\section{- Normalization of RET}

In this paper, the link stability is characterized by the parameter of RET. At instant $t$, let $\left(x_{s}, y_{s}\right)$ and $\left(x_{i}, y_{i}\right)$ denote the position of $\mathrm{SV}$ and $i$ th candidate RS, respectively, $r$ denotes the maximum transmission range of the candidate RV. The LET of the link between SV and the $i$ th candidate RV can be expressed as [14]:

$$
L E T_{S, V_{i}}=\frac{-(a b+c d)+\sqrt{\left(a^{2}+c^{2}\right) r^{2}-(a d-b c)^{2}}}{a^{2}+c^{2}}
$$

Where, $a=v_{i} \cos \theta_{i}-v_{s} \cos \theta_{s}, b=x_{i}-x_{s}, c=v_{i} \sin \theta_{i}-v_{s} \sin \theta_{s}$, $d=y_{i}-y_{s}$.
The LET between the $i$ th RV and RSU can be calculated as:

$$
L E T_{V_{i}, R}=\frac{d_{V_{i}, R} \cos \theta_{V_{i}, R}+\sqrt{R_{0}^{2}-d_{V_{i}, R}^{2} \sin ^{2} \theta_{v_{i}, R}}}{v_{i}}
$$

Where $R_{0}$ denotes the maximum wireless transmission range of RSU, $\theta_{r_{i, R}}$ denotes angle between the connection line of the $i$ th RV and RSU and the moving direction of $i$ th RV.

Hence, the RET of the route between SV and RSU can be expressed as:

$$
R E T_{i}=\min \left(L E T_{S, V_{i}}, L E T_{V_{i}, R}\right)
$$

Normalization of RET can be expressed as:

$$
l_{i 3}^{V}=\frac{R E T_{i}}{\max \left(R E T_{1}, R E T_{2}, \ldots, R E T_{N}\right)}
$$

\section{Attribute Weighting}

The attribute weight is used to reflect the relative importance of each attribute, the attribute more important, the greater the weight. In general, MADM model requires the weight information based in a basic scale. Basic weights are expressed by $\omega=\left[\omega_{1}, \omega_{2}, \ldots, \omega_{j}, \ldots, \omega_{n}\right]$, where $\omega_{j}$ is the weight of $j$ th attribute. Hence, basic weights are normalized to 1 expressed as $\sum \omega_{j}=1$.

3. Scheme ranking comprehensive

Gather the decision information by some way, sort and select the optimal scheme. In this paper, simple additive weighting is used. So the utility function can be express as:

$$
Q_{i}^{V}=w_{B} l_{i 1}^{V}+w_{D}\left(1-l_{i 2}^{V}\right)+w_{R} l_{i 3}^{V}
$$

According to (16), the candidate RV with the highest $Q_{i}^{V}$ should be chosen as the destination RV, namely:

$$
i^{*}=\arg \max _{i}\left(Q_{i}^{V}\right), \quad i=1,2, \ldots, N
$$

\section{Numerical example}

In the section, the performance of the proposed RV selection algorithm is evaluated in MATLAB, and compared with previous algorithm proposed in [4]. We consider a straight road of $1 \mathrm{Km}$, where vehicles (source vehicles and RV vehicles) are located randomly and one RSU placed in the middle of road. The electric vehicles move along the road with the random velocity varied from $80 \mathrm{Km} / \mathrm{h}$ to $120 \mathrm{Km} / \mathrm{h}$. The number of total vehicles range from 20 to 60 . We choose the number of SV and RV being 20 and 40, respectively.

Figure 2 shows the average throughput of all the vehicles versus the simulation time. It can be seen from the figure that the proposed scheme is superior to the maximum LET selection algorithm in average throughput. This is because the date transmission rate of candidate $\mathrm{RV}$ is chosen as an important factor for proposed algorithm, result a relatively higher throughput. 
The transmission delay of various algorithms is given in Figure 3. It can be seen from the figure, the transmission delay increase with the increase of the number of vehicle. The reason is that the probability of collision and packet retransmission times increase for larger vehicle numbers. Compared with maximum LET algorithm, the proposed algorithm has lower average delay.

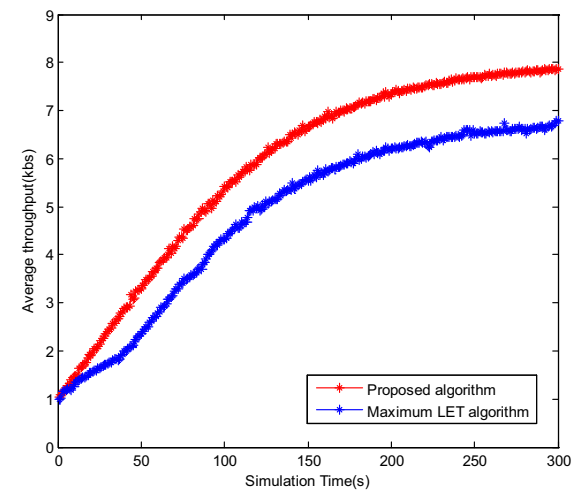

Figure 2 Average throughput

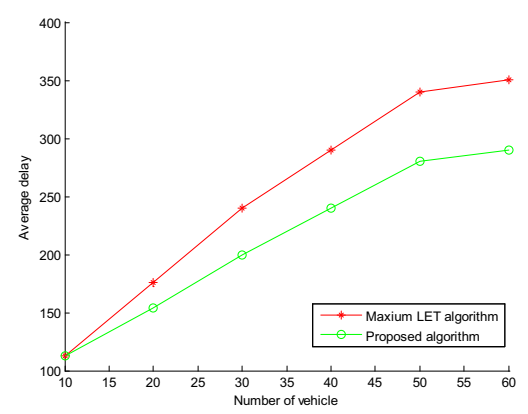

Figure 3 Average transmission delay

\section{Conclusion}

In this paper, a multiple attribute decision making theory based RV selection algorithm is proposed for the information transmission network electric vehicle to RSU. We choose three attributes: data transmission rate, delay, the stability between source vehicle and RSU via RV, and apply simple additive weighting method for evaluating the performance of available RSs. Simulation results demonstrate that the proposed scheme offers better average throughput and average delay of source vehicles.

\section{Acknowledgment}

This work was supported by the National Natural Science Foundation of China under Grant No.61104005 and 61374097, by Natural Science Foundation of Liaoning Province under Grant No.201202073, and by the Open Research Fund from the State Key Laboratory of Rolling and Automation, Northeastern University, Grant No. 2015001 .

\section{References}

1. D. Streimikiene, T. Baležentis, L. Baležentienè, Comparative Assessment of Road Transport Technologies, General Information, 20, 4(2013)

2. A. Hess, F. Malandrino, MB Reinhardt, Optimal deployment of charging stations for electric vehicular networks, Proceedings of First ACM CONEXT Workshop on Urban Networking, (2012)

3. A. Boukerche, H.A.B.F. Oliveira, E. Nakamura,

A.A.F. Loureiro, Vehicular Adhoc networks: A New Challenge for Localization-Based Systems, Computer Communications, 31, 12(2008)

4. A. Benslimane, S. Barghi, C. Assi, An Efficient Routing Protocol for Connecting Vehicular Networks to the Internet, Pervasive \& Mobile Computing, 7, 1(2011)

5. T. P. Van, V. D. Nguyen, Location-aware and Loadbalanced Data Delivery at Road-side Units in Vehicular Ad hoc Networks, IEEE International Symposium on Consumer Electronics, 2010

6. Y. Ge, S. Wen, Y. Ang, et al, Optimal Relay Selection in IEEE 802.16j Multihop Relay Vehicular Networks, IEEE Transactions on Vehicular Technology, 59, 5(2010)

7. H. Khayatian, R. Saadat, and J. Abouei, Coalitionbased Approaches for Joint Power Control and Relay Selection in Cooperative Networks, IEEE Trans. Veh. Technol., 62, 2( 2013)

8. H. Ilhan, M. Uysal, and I. Altunbas, Cooperative Diversity for Inter Vehicular Communication: Performance Analysis and Optimization, IEEE Trans. Veh. Technol., 58, 7(2009)

9. G. K. Karagiannidis, N. C. Sagias, and P. T. Mathiopoulos, N*Nakagami: A novel stochastic model for cascaded fading channels, IEEE Trans. Communications, 55, 8(2007)

10. I.S.Gradshteyn, I.M.Ryzhik, Table of Integrals, Series, and Products, seventh edition, Draft of Latex Class Files, 2000.

11. T. S. Rappaport, Wireless Communications, Principle and Practice. Prentice Hall, 2002

12. J.Butler, D.J. Morrice, P.W.Mullarkey, A Multiple Attribute Utility Theory Approach to Ranking and Selection, Management Science, 47, 6(2001)

13. Y. Paul and H. Ching-Lai, Multiple Attribute Decision Making: An Introduction, Sage Publications, 1995.

14. A. Benslimane, S. Barghi, C. Assi, An Efficient Routing Protocol for Connecting Vehicular Networks to the Internet, Pervasive \& Mobile Computing, 7, 1(2011) 